\section{Social and Emotional Learning Program Among Preschool Children}

Social and emotional learning (SEL) is the process through which children and adults understand and manage emotions, set and achieve positive goals, feel and show empathy for others, establish and maintain positive relationships, and make responsible decisions [1]. Currently, in India, SEL is not being given importance due to changing lifestyle, urbanization, both parents working, single child, and non-availability of secure play areas etc. Children are sent to preschool early, with a focus on academics rather than on personal development. This makes them socially and emotionally detached [2]. We share our experience of a short duration SEL program at kindergarten level.

Children aged 5-6 years, studying in upper kindergarten were enrolled in the program, and data of those with attendance less than $80 \%$ in the SEL program was excluded. An introductory class was taken for the parents about SEL. School administrators, teachers and parents were actively involved throughout the sessions. Eleven hours of the program was conducted over 6 weeks ( 1 hour session, 2 per week, conducted by a trained psychologists). Children were given work sheets and fun activities as home project to ensure parental participation. Questionnaires based on the social skill improvement system were administered to the parents before and after the sessions to see the effect of the program. School was given inputs to integrate SEL into the curriculum. Teachers' comments were also taken into consideration to identify any problem behaviors. The SSIS (Social skill improvement system) parent form (social skills subdomain, 3-5 years) was used to provide a picture of child's behavior in multiple settings. However, due to the large number of students to teacher ratio (40:1), the teacher form was not included. The Kimochis Educator's Tool Kit was used. It is a universal, school-based, social emotional learning curriculum designed to give children the knowledge, skills and attitudes they need to recognize and manage their emotions.

113 children ( 58 boys; mean age, 5.3 years) were included in the study, and classified to two groups based on the behavior levels obtained from the pre-SEL questionnaire. Group 1 $(n=23)$ had below average and well below average level on social skills sub scale, and children in group $2(n=90)$ had an average, above average and well above average level of social skills. The mean score for each of the social skill sub-domain raw scores (communication, cooperation, assertion, responsibility, empathy, engagement and self-control) and total score was calculated for groups. Analysis of variance (ANOVA) was used to compare scores between the groups. Analysis was done using Microsoft Excel. A 5\% level of significance was considered significant.

In Group 1, 15 (65.2\%) children showed an improvement in the total social skills raw score following the intervention. There was a significant improvement in mean (SD) total social skills score after the intervention [64.7 (8.16) vs. 72.1 (10.74); $\mathrm{P}<0.01]$, indicating short term Kimochi training is effective for children. In Group B, 40 showed an improvement in the total social skill score and 47 (44.4\%) children showed reduction in scores. No statistically significant difference was noted in the pre-and post-intervention mean (SD) total skill score in this group [91.3 (14.26) vs $90.8(16.21) ; P=0.82]$.

However, follow-up is needed to see the impact of the skills learned, as children need time to practice and integrate the learned skills (sleeper effect) [3]. The CASEL meta-analysis [4] noted that though impact is maximum immediately, it still persists, though the impact of the program tends to fade.

At the closing session of our program, parents were advised how to promote SEL at home. Children with problem behaviors were guided appropriately. Preschool teachers' workshop was conducted to promote professional development and early identification of developmental problems. We feel that our experience shall promote more studies to establish healthy SEL at schools, especially in the Indian scenario, where there is a paucity of the same.

Acknowledgement: Dr Nandini Mundkur, Developmental Paediatrician, Center for Child Development and Disabilities, Bengaluru, Karnataka, India.

\section{AKHIla NagaraJ* and Kirthika RaJaraman Center for Child Development and Disabilities, Malleswaram, Bengaluru, Karnataka. *drakhilanagaraj@yahoo.com}

\section{REFERENCES}

1. The Collaborative for Academic, Social, and Emotional Learning (CASEL). Accessed March 20, 2021. Available from: https:// casel.org/

2. Rajawat D, Patel RC. Identifying the tenets for socio-emotional learning (SEL) and development of pre-schoolers. ISOR - JRME. 2015;5:25-38

3. Blewitt C, Fuller-Tyszkiewicz M, Nolan A, et al. Social and emotional learning associated with universal curriculum-based interventions in early childhood education and care centre: A systematic review and meta-analysis. JAMA Network Open. 2018;1: e185727.

4. Mahoney J, Durlak J, Weissberg RP. An update on social and emotional learning outcome research, 2018. Accessed March 20, 2021. Available from: https://kappanonline.org/social-emotional-learning-outcome-research-mahoney-durlak-weissberg/ 